\section{New Delhi Metallo- $\beta$ - Lactamase-Producing Enterobacterales Bacteria}

\author{
Yancheng Yao, Can Imirzalioglu, Linda Falgenhauer, \\ SurvCARE Hesse Working Group, Trinad Chakraborty
}

Author affiliation: Justus Liebig University German Center for Infection Research, Giessen, Germany.

DOI: https://doi.org/10.3201/eid2801.212106

To the Editor: Findlay et al. (1) reported a high prevalence $(>25 \%)$ of New Delhi metallo- $\beta$-lactamase (NDM) among carbapenemase-producing Enterobacterales (CPE) in Switzerland during 2019-2020. To provide context, we analyzed recent trends for NDMproducers in Germany.

In a whole-genome-sequencing-based surveillance study conducted in 52 hospitals in Hesse, Germany, during 2017-2019, we detected 56 NDM-producing isolates among 346 CPE. The prevalence of NDM CPE increased from 6.3\% (5/79) in 2017 to $15.0 \%(17 / 113)$ in 2018 and $22.1 \%(34 / 154)$ in 2019. A total of 56 alleles - 28 NDM-1, 27 NDM-5, and 1 NDM-7 - were detected in 31 Klebsiella pneumoniae, 18 Escherichia coli, and 3 Citrobacter freundii isolates and 1 isolate each of C. portucalensis, Enterobacter hormaechei, K. grimontii, and K. variicola. Isolates were derived from rectal swab (30), urine (11), respiratory tract (6), and other (9) specimens. More than 82\% (23/28) of NDM-1 were associated with K. pneumoniae and 59\% $(16 / 27)$ of NDM-5 with E. coli; $41 \%$ of the NDM-CPE harbored a 16S-rRNA-methylase gene.

Among carbapenemase-producing K. pneumoniae isolates, NDM increased from 6.4\% (3/47) in 2017 to $24.5 \%(13 / 53)$ in 2018 and $31.9 \%(15 / 47)$ in 2019; $\approx 50 \%(15 / 31)$ were sequence type 147 . Thus, reports for Switzerland (1) and Italy (2) indicate that sequence type 147 NDM-carrying K. pneumoniae is a CPE clone rapidly emerging in Europe.

NDM accounted for $20 \%(2 / 10)$ of carbapenemase-producing E. coli in 2017, 11.1\% (2/18) in 2018, and $24.1 \%(14 / 58)$ in 2019 ; the increase in NDM5-producing E. coli was primarily associated with
IncF-type plasmids (3). A subgroup harboring penicillin binding protein 3 modifications exhibited increased aztreonam/avibactam MICs of $2-8 \mu \mathrm{g} / \mathrm{mL}$. Higher MICs $(4-16 \mu \mathrm{g} / \mathrm{mL})$ were associated with the presence of an additional highly conserved $b l a_{\mathrm{CMY}-42^{-}}$ encoding IncIY plasmid (4). We reiterate the call by Findlay et al. to implement genome-based surveillance studies to identify emerging clonal lineages and commonly occurring plasmids among carbapenemase-producing bacteria.

\section{Acknowledgments}

We thank members of the SurvCARE Hesse Working Group who contributed data or sample collection or both: Eugen Domann, Jane Falgenhauer, and Moritz Fritzenwanker (Justus Liebig University, Giessen, Germany); Petra Heinmüller and Gudrun Bettge-Weller (Hessisches Landesprüfungs und Untersuchungsamt im Gesundheitswesen, Dillenburg, Germany); and Anja M. Hauri (Helmholtz Centre for Infection Research, Braunschweig, Germany).

\section{References}

1. Findlay J, Poirel L, Kessler J, Kronenberg A, Nordmann P. New Delhi metallo- $\beta$-lactamase-producing Enterobacterales bacteria, Switzerland, 2019-2020. Emerg Infect Dis. 2021;27:2628-37. https://doi.org/10.3201/ eid2710.211265

2. Tavoschi L, Forni S, Porretta A, Righi L, Pieralli F, Menichetti F, et al. Prolonged outbreak of New Delhi metallo-betalactamase-producing carbapenem-resistant Enterobacterales (NDM-CRE), Tuscany, Italy, 2018 to 2019. Vol. 25, Eurosurveillance. European Centre for Disease Prevention and Control (ECDC); 2020.

3. Chakraborty T, Sadek M, Yao Y, Imirzalioglu C, Stephan R, Poirel L, et al. Cross-border emergence of Escherichia coli producing the carbapenemase NDM-5 in Switzerland and Germany. J Clin Microbiol. 2020; 59:e02238-20.

4. Nordmann P, Yao Y, Falgenhauer L, Sadek M, Imirzalioglu C, Chakraborty T. Recent emergence of aztreonam-avibactam resistance in NDM and OXA-48 carbapenemase-producing Escherichia coli in Germany. Antimicrob Agents Chemother. 2021;65:e0109021. https://doi.org/10.1128/AAC.01090-21

Address for correspondence: Trinad Chakraborty, Institute of Medical Microbiology, Justus Liebig University Giessen, Schubertstrasse 81, D-35392 Giessen, Germany; email: trinad. chakraborty@mikrobio.med.uni-giessen.de

\title{
Correction: Vol. 27, No. 9
}

The acknowledgments and funding information were inaccurate in Severe Acute Respiratory Syndrome Coronavirus 2 in Farmed Mink (Neovison vison), Poland (L. Rabalski et al.). The article has been corrected online (https:/ / wwwnc.cdc.gov/eid/article/27/9/21-0286_article). 